Research Article

\title{
Understanding Friction and Wear Behaviours of Smooth Resilient Surfaces: Application for Pedestrian Fall Safety Improvements
}

\author{
In-Ju Kim (i) \\ Department of Industrial Engineering and Engineering Management, College of Engineering, University of Sharjah, \\ P.O. Box 27272, Sharjah, UAE \\ Correspondence should be addressed to In-Ju Kim; dr.injukim@gmail.com
}

Received 3 May 2018; Accepted 24 September 2018; Published 16 October 2018

Academic Editor: Pietro Russo

Copyright @ 2018 In-Ju Kim. This is an open access article distributed under the Creative Commons Attribution License, which permits unrestricted use, distribution, and reproduction in any medium, provided the original work is properly cited.

The recent literature identified that certain types of smooth floors and flooring materials showed good slip-resistance performance under moderately contaminated situations such as water wet and soapsuds-covered conditions. However, topographic structures of such flat floor/walkway surfaces are significantly changed by wear and tear developments with continuous ambulation. A number of reasons seem to be involved in flooring wear developments, but such changes may result in substantial losses of slip resistance (or traction) functions. Even though the importance of this issue is wide, it is scarce to find any systematic investigations on tribophysical characteristics of smooth floors $\left(<10 \mu \mathrm{m}\right.$ in $R_{\mathrm{a}}$ roughness) and their wear impacts on traction performance. Moreover, it is difficult to find studies on wear progress of floors and its impacts on pedestrian fall safety assessments. This study focuses on exploring wear activities of smooth resilient floors and disclosing wear consequences on traction properties. To measure slip-resistance features and investigate wear advances of smooth resilient floors, dynamic friction tests were carried out amongst purposely arranged 4 flat specimens (polymethyl methacrylate: PMMA) and 3 shoes. Wear formations and progressions of the PMMA surfaces were methodically evaluated by observing surface profiles during the tests. The test outcomes evidently presented that initial surface features of the PMMA specimens were significantly modified by primary-, secondary-, and sharedwear mechanisms and significantly influenced traction performance. This study may have design applications for the safety enhancements of smooth floors/footpaths to preclude pedestrian fall incidents.

\section{Introduction}

Pedestrian pavements and floors must be well-located and deliver safe environments at all levels of physical activities and events. They should be accessible and offer comfortable and pleasurable walking experiences. They are also likely to largely affecting the outputs and operations of industries and organizations [1]. Thus, floors and floor coverings should be constructed to deliver safe ambulatory conditions and right slip-resistance functions under any slick situation. To achieve those goals, they have to be cautiously prearranged and assessed as they comprise performance conditions for construction resources and are a primary issue for the design of floors [2].

Because abundant choices of building materials and products for walkways and footpaths are offered on the market, choosing the best floors and floor coverings for a particular business or industry category would be one of the most difficult missions to determine safety and stability over time periods [1]. Amongst a variety of flooring choices, a smooth floor and floor covering such as vinyl is one of the most affordable resilient building materials and solutions accessible [3]. Because of versatility, low maintenance, high durability, comfort underfoot, and more moisture-resistant features than many alternative materials, vinyl and vinyl materials turn out to be a popular flooring choice [4]. Vinyl floorings also become a valued design tool for many interior designers and architects, particularly those devising for heavy-traffic places [5]. For example, hospitals, nursing homes, commercial kitchens, and light industrial premises are popular areas for the usage of vinyl floorings. Regardless of the advantages of material characteristics, versatilities, low maintenance, and popularities, vinyl floorings are susceptible to chips, warping, and scratches. Such damages can 
begin to appear from the stage of initial installation, especially in high travelling areas so extra care is required to maintain the aesthetics.

Another challenging concern is about controlling and maintaining texture conditions of the floor surfaces because they seem to be continuously modified by abundant reasons such as maturing, erosion, fouling, and sustaining $[1,6]$. Accordingly, gradual wear and damage progress are inevitable and may significantly impact on traction functions [7-10]. This issue was reported by several studies in the views of profile roughness, textures, and friction properties [1117]. However, inclusive investigates on flooring wear behaviours and effects on pedestrian fall safety assessments are scared to find in the literature [18-21]. In particular, flooring wear mechanisms and impacts on skid resistance performance were not methodically explored [1, 7-10]. The recent literature highlighted that smooth surfaces $(<10 \mu \mathrm{m}$ in $R_{\mathrm{a}}$ roughness) showed good slip-resistance functioning against dry and moderately lubricated surroundings such as water wet and soapsuds-covered foamy situations $[7,9,10]$. Functional ranges of the floor surface roughness signified that coarser floors and/or coarsening surfaces would not always require enhancing slip-resistance properties $[7,9,10]$. Hence, it becomes evident that walkways need disparate roughness for diverse walking environments to manage walkway slipperiness successfully. Those findings clearly suggest that traction properties of the smooth floors need advance investigations to comprehend their tribological features and impacts on traction functioning.

The purpose of the present study is to identify friction and wear developments of smooth floors and recognize their consequences on traction functioning. The main theoretical approach was based on the previous study for flooring wear behaviours and a paradigm to explore friction and wear mechanisms of smooth metal specimens with slip-resistance measurements [1]. Although the flooring wear model was developed from hard metal materials, the current study is intended to verify the model with totally different types of flooring material such as resilient ones.

To observe wear activities and progresses of the smooth resilient floor surfaces with slip-resistance properties, laboratory-based skid resistance tests were executed amongst deliberately arranged 4 polymethyl methacrylate (PMMA or acrylic) sheets and three shoes. Selection of the PMMA specimens was projected to eliminate any commercial type of floorings and floor materials, provide objective measures of frictional properties, and identify wear behaviours of vinyl-type flooring materials during the tests. Original and tested surfaces of the PMMA samples were expansively analyzed by measuring surface profiles and observing surface structures to recognize wear behaviours and growths before and after the tests. For the observation of surface structures, a scanning electron microscope (SEM) was operated to detect structure alterations and wear advances of the PMMA samples before and after the tests.

Overall outcomes confirmed the wear model for floor surfaces suggested in the previous study $[1,8]$ and attested that a series of primary- and secondary-wear mechanisms were mainly caused to wear evolutions of the smooth resilient surfaces. The significance of flooring wear advances due to repeated friction events and impacts on skid resistance controls were clearly recognised as well. Findings from this study may apply for the design enhancements and the development of new flooring products for walkways and footpaths to avoid pedestrian fall incidents.

\section{Materials and Methods}

2.1. Floor and Shoe Samples. To impartially measure slipresistance properties and understand tribophysical characteristics of vinyl-type flooring surfaces, polymethyl methacrylate (PMMA or acrylic) sheets were deliberated for dynamic friction measurements. The PMMA sheets were arranged by a shot-blasting method to create dissimilar surface texture. 4 changed levels of blasting intensities and durations were applied to clean PMMA sheets (dimension: $11.0 \times 17.0 \mathrm{~cm})$. The shot-blasted PMMA surfaces were airbrushed to eliminate any fine dry particles and maintained in glass containers for the tests. After the flooring samples were prepared, they were labelled as PMMA1 to $P M M A 4$ according to their coarseness scales. Figure 1 shows photographic images of the finished surfaces of PMMA sheets before the tests.

For shoe samples, 3 new shoes: polyurethane, PVC, and nitrile rubber were employed for the tests. They had different hardness scales and heel/sole types: polyurethane (50 Shore A Hardness), PVC (65 \pm 5 Shore A Hardness), and nitrile rubber (70 \pm 5 Shore A Hardness). Each shoe sole/heel was fully cleaned with demineralised waters and stored in plastic containers for the tests.

2.2. Dynamic Friction Tester and Test Conditions. Dynamic friction tests were executed by using a pendulum-type hydraulic friction device $[1,7,8]$. This device was developed to emulate foot movements during ambulatory actions such as heel strikes and initial slips. It can measure skid conditions as a coefficient of dynamic friction (DFC) $[1,7,8]$. A loading force was retained about $350 \mathrm{~N}$ and a skidding speed was operated at $40 \mathrm{~cm} / \mathrm{sec}$ in accordance with walking tests $[1,4,5]$. A shoe strike angle of $9^{\circ}$ was also designated by the earlier walking trials $[1,7,8]$.

The tests were performed under clean and dry environments to remove any compounding influences rather than floor and shoe specimens themselves. This controlled test condition was intended to investigate topographic features of the PMMA surfaces by repeated sliding frictioninduced wear developments and identify their controls on skid resistance functioning during the tests.

The skid-resistance tests were also conducted from the flattest surface (PMMA1) to the coarsest one (PMMA4) along with the roughness levels of variations in the PMMA specimens. This test arrangement was deliberate to investigate wear advances of the PMMA surfaces and lessen early ravages on the PMMA ones during the tests. The shoe specimens were also arranged by the order of shoe hardness scales: Polyurethane shoe (50 Shore A Hardness) to nitrile rubber one (70 \pm 5 Shore A Hardness). 


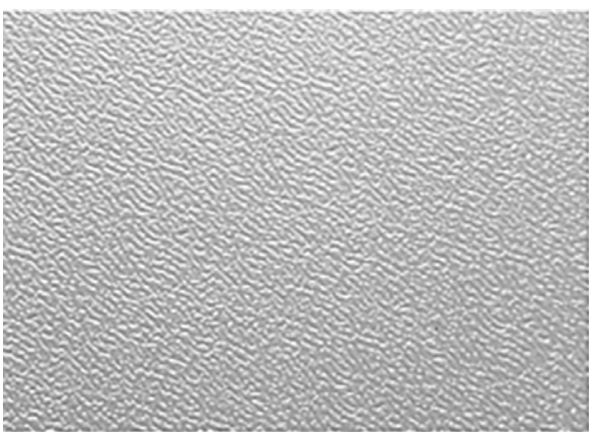

(a)

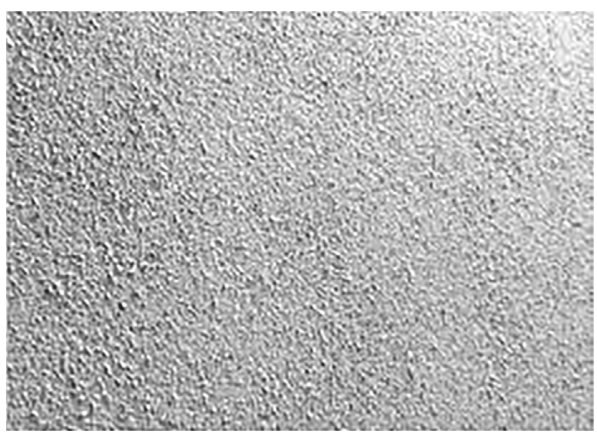

(c)

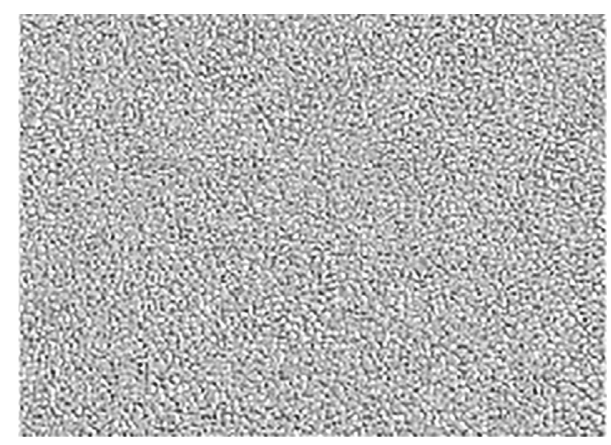

(b)

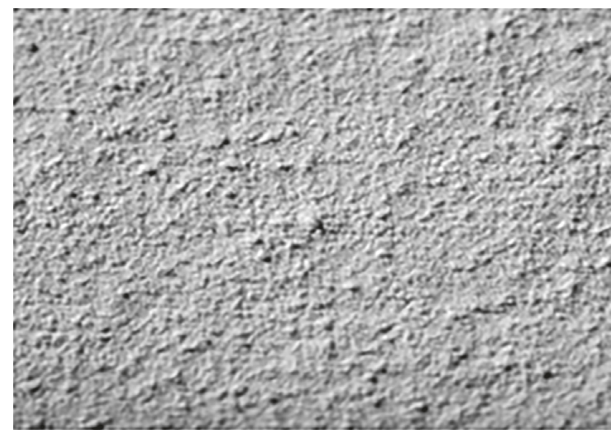

(d)

FIGURe 1: Photographic images of the four PMMA sheets prepared by shot-blasting techniques before the dynamic friction tests: (a) PMMA1, (b) PMMA2, (c) PMMA3, and (d) PMMA4.

Each floor-shoe pair was tested by two stages to observe surface changes and wear progress of the PMMA surfaces. The three shoes were tested 10 times against the PMMA surfaces and retested another 10 times against the rubbed PMMA ones. Both floor and shoe surfaces were carefully dusted with a soft brush to eliminate freed particles after a single skidding.

2.3. Measuring Surface Profiles. A laser scanning confocal microscope (LSCM, Bio-Rad MRC-600) was employed to quantify the profile roughness of PMMA specimens before and after the tests [22]. Figure 2 shows a photographic image of the LSCM used for this study. The images of $x-z$ axial "slices" were created by a sequence of parallel line scans, whilst the sample was stepped by perpendicularly in the $z$ direction with a step size of $0.1 \mu \mathrm{m}$ [22].

The surface roughness of each PMMA specimen was assessed three times at five different locations, and its mean values were adopted for the further investigations. The profile roughness parameters were chosen by findings from the previous studies $[1,7-10,23,24]$. Full information on the surface roughness parameters is uncovered in the published studies [25, 26]. Table 1 outlines the final surface conditions and roughness features of each PMMA sheet before the tests. The measured roughness parameters present topographic structures of the resultant surfaces for each PMMA specimen.

2.4. Observing Surface Structures. A stereo scanning electron microscope (SEM: XL 30, Philips) was used to cautiously examine the surfaces of PMMA samplings before and after the tests. The microscopic works were also proposed to support profile roughness data, recognize wear mechanisms, and detect wear progress of the PMMA surfaces after the friction tests.

\section{Results and Discussion}

\subsection{Results}

3.1.1. Slip-Resistance Performance. Figure 3 displays results of skid-resistance performance amongst the 4 PMMA samples and 3 shoes. The DFCs were plotted against the surface roughness ( $R_{\mathrm{a}}$ parameter) of four PMMA specimens between the two stages of dynamic friction tests. As found in Figure 3 , the DFCs were largely changed after the two stages of tests except for the case of the PVC-PMMA groups.

All the PMMA-shoe combinations disclosed high traction performance (DFCs $>0.8$ ) but showed different slipresistance trends. For example, after the first ten rubbings (first test), the performance of slip resistance showed the following rank order: polyurethane $>$ nitrile rubber $>$ PVC shoes against the PMMA surfaces. However, the slipresistance performance presented a different rank order: $\mathrm{PVC}>$ polyurethane $\approx$ nitrile rubber after the second ten rubbings (second test).

The DFCs were clearly decreased by $23 \%$ to $28 \%$ for the polyurethane shoe and about $5 \%$ for the nitrile rubber one against each PMMA surface, respectively. In contrast, the DFCs of the PVC shoe were largely improved by 15 to $37 \%$ against each PMMA surface. 


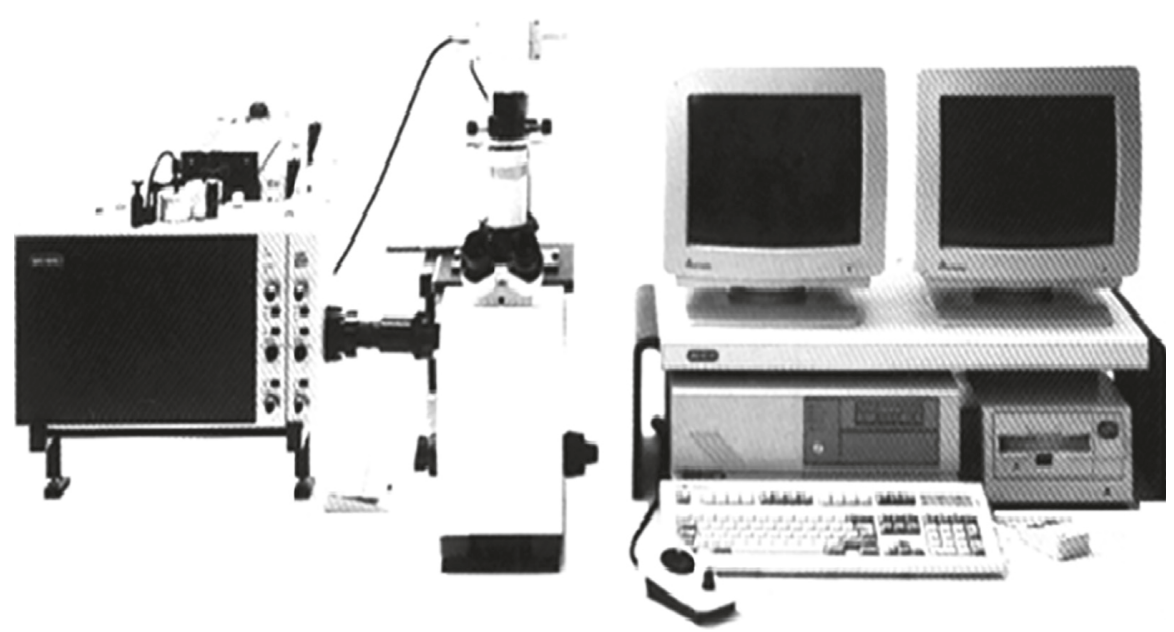

Figure 2: The photographic image of the laser scanning confocal microscope (LSCM, Bio-Rad MRC-600) used for this study.

TABLE 1: Measurement results of the surface roughness parameters for the PMMA specimens before (BDFT) and after the dynamic friction tests (ADFT).

\begin{tabular}{|c|c|c|c|c|c|c|c|c|c|}
\hline \multirow{2}{*}{ Test condition and changing rate } & \multirow{2}{*}{$\begin{array}{l}\text { PMMA } \\
\text { specimen }\end{array}$} & \multicolumn{8}{|c|}{ Surface roughness parameter $(\mu \mathrm{m})$} \\
\hline & & $R_{\mathrm{a}}{ }^{1}$ & $R_{\mathrm{q}}^{2}$ & $R_{\mathrm{tm}}^{3}$ & $R_{\mathrm{pm}}^{4}$ & $R_{\mathrm{vm}}^{5}$ & $\Delta_{\mathrm{a}}{ }^{6}$ & $R_{\mathrm{sk}}{ }^{7}$ & $R_{\mathrm{kr}}^{8}$ \\
\hline \multirow{4}{*}{ BDFT } & PMMA1 & 2.330 & 3.121 & 16.817 & 6.483 & -7.492 & 0.072 & -0.432 & 1.002 \\
\hline & PMMA2 & 3.769 & 4.756 & 22.316 & 8.968 & -9.483 & 0.096 & -0.256 & -0.011 \\
\hline & PMMA3 & 4.213 & 5.212 & 23.299 & 8.930 & -10.968 & 0.102 & -0.202 & -0.242 \\
\hline & PMMA4 & 4.837 & 6.082 & 27.800 & 11.745 & -11.175 & 0.122 & -0.046 & 0.180 \\
\hline \multirow{4}{*}{ ADFT } & PMMA1 & 1.477 & 1.973 & 15.860 & 4.882 & -3.645 & 0.041 & 0.283 & 1.624 \\
\hline & PMMA2 & 1.895 & 2.543 & 16.829 & 5.640 & -4.303 & 0.059 & 0.295 & 2.804 \\
\hline & PMMA3 & 2.190 & 3.033 & 20.307 & 6.204 & -5.236 & 0.069 & 0.280 & 2.569 \\
\hline & PMMA4 & 2.933 & 3.807 & 23.293 & 6.438 & -6.037 & 0.078 & 0.248 & 1.344 \\
\hline \multirow{6}{*}{$\begin{array}{l}\text { Changing rate of surface roughness } \\
\text { parameters }(\%)\end{array}$} & PMMA1 & 36.61 & 36.78 & 5.69 & 24.7 & 51.35 & 43.06 & & \\
\hline & PMMA2 & 49.72 & 46.53 & 24.59 & 37.11 & 54.62 & 38.54 & & \\
\hline & PMMA3 & 48.02 & 41.81 & 12.84 & 30.53 & 52.26 & 32.35 & $-0.234^{9}(\mathrm{BDFT})$ & $0.232^{10}(\mathrm{BDFT})$ \\
\hline & PMMA4 & 39.36 & 37.41 & 16.21 & 45.19 & 45.98 & 36.07 & $0.227^{11}(\mathrm{ADFT})$ & $2.085^{12}(\mathrm{ADFT})$ \\
\hline & Avg. & 43.43 & 40.63 & 14.83 & 34.38 & 51.05 & 37.51 & & \\
\hline & Std. dev. & 6.42 & 4.52 & 7.85 & 8.81 & 3.65 & 4.49 & & \\
\hline
\end{tabular}

1: centre-line average roughness; 2 : root-mean-square roughness; 3 : maximum mean peak-to-valley height; 4: maximum mean peak height; 5 : maximum mean depth height; $6 . \Delta_{\mathrm{a}}$ : average slope angle; 7 : skewness; 8 : kurtosis; 9: average of the initial surfaces' skewness; 10: average of the rubbed surfaces' skewness; 11: average of the initial surfaces' kurtosis; 12: average of the rubbed surfaces' kurtosis.

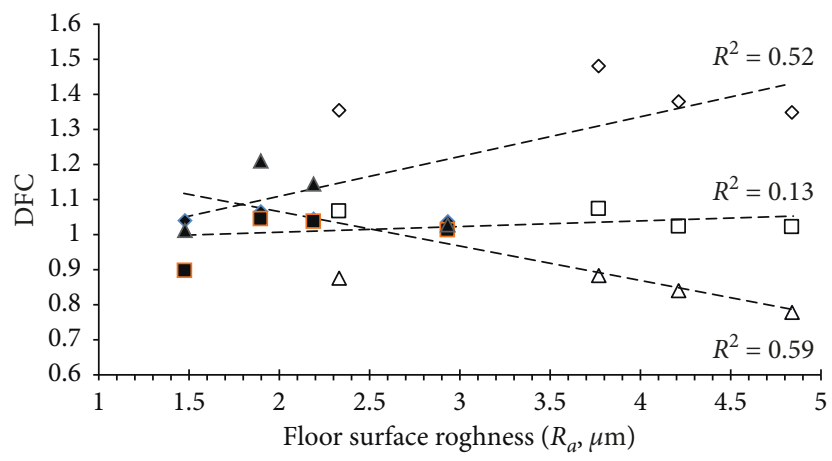
(1) First test
(2) Second test
$\diamond$ Polyurethane
- Polyurethane
$\square$ Nitrile rubber
- Nitrile rubber
$\triangle \mathrm{PVC}$
^ PVC

FIGURE 3: A result of dynamic skid-resistance measurements between the four PMMA specimens and three shoes: (1) average of first ten rubbings and (2) average of second ten rubbings, respectively. 
3.1.2. Wear Evaluations: Measuring Surface Profiles. Table 1 presents the consequences of profile roughness parameters of the PMMA samplings. As clearly shown in Table 1, all the profile roughness parameters underwent large changes from the initial states to the rubbed ones. $R_{\mathrm{vm}}$ (maximum mean depth height) was significantly decreased by over $51 \%$ after the tests. This result showed the largest reduction amongst the assessed profile roughness parameters from this study. On the contrary, $R_{\mathrm{tm}}$ roughness parameter (maximum mean peak-to-valley height) presented the least change (average 14.83\%) after the tests. As also found in Table 1, the initial PMMA surfaces had fairly negative profiles indicating the predominance of valley areas. After the tests, $R_{\mathrm{sk}}$ and $R_{\mathrm{kr}}$ of PMMA surfaces were largely transformed into positively skewed ones (on average from -0.234 to 0.227 for $R_{\mathrm{sk}}$ and from 0.232 to 2.085 for $R_{\mathrm{kr}}$, respectively).

\subsubsection{Wear Evaluations: Observing Surface Structures}

(1) Wear Observation. As shown in Figure 1, the micrographs for each PMMA specimen visibly revealed that a number of small indented asperities covered the initial surfaces thus produced negatively skewed topographic structures. These surface features seemed to be caused by the shot-blasting process to roughen the PMMA specimens. After the tests, however, the PMMA surfaces experienced extensive wear developments.

Figure 4 shows the sample photoelectron micrographs for the PMMA surfaces after the tests. Major outcomes on wear behaviours of the PMMA surfaces are condensed as follows:

(1) Bulky amounts of wear products were found on the tested surface trails, depending upon the profile roughness of the PMMA samples

(2) Repetitive rubbings caused to the relocation of polymer fragments from the shoe surfaces to the PMMA ones

(3) Wear transfers were frequently discovered in the wide valley regions of the PMMA surfaces

(4) Irregular-shaped and dissimilar-sized wear particles were often detected in the cracked and wedged surface regions of PMMA samples

(5) Relocated wear debris and particles did not severely sheath the top asperities of PMMA surfaces

(2) Wear Fragments and Particles. Figure 5 shows instances of wear products formed at top asperities and basin regions of the PMMA surfaces after the tests. The major disparities in wear behaviours amongst the four PMMA samples appear to be instigated by two key causes: original roughness features of the PMMA surfaces and rubbed shoe classes. Most of the wear products are found in the forms of uneven shapes and patterns, and the diverse sizes of fragments and particles which fill the asperity crevices in a number of different locations.

\subsection{Discussion}

3.2.1. Observation of Slip-Resistance Performance. Measured DFC outcomes amongst the 4 PMMA samples and 3 shoes showed high skid-resistance functioning during two sets of dynamic friction measurements (Figure 3). However, there were different developments in the overall slip-resistance operations. For example, as found in Figure 3, the PMMA2 $\left(R_{\mathrm{a}}=3.769 \mu \mathrm{m}\right)$ presented the highest slip resistance than the other three PMMA specimens against all the three shoes. This result indicated that there were strong interfacial interactions amongst the PMMA2 surface and three shoes than any other shoe-PMMA combination.

It was also found that the DFCs between the PMMA4 $\left(R_{\mathrm{a}}=4.837 \mu \mathrm{m}\right)$ and three shoes showed a converging fashion after the test (Figure 3 ). This consequence signified that heavy growths of early wear behaviours were settled down at the PMMA4. This emerging trend was also observed in the recent studies which measured DFCs amongst the four metal samples and three shoes but was even stronger in the case of PMMA surfaces than the metal ones. Because the PMMA specimens have a lower elastic modulus than the general flooring material types including metals, there seems to be a strong possibility that plastic deformations have rather severely occurred in the PMMA surfaces than the case of metal ones. This aspect of wear behaviours needs advance investigations to fully realize their mechanisms and prolonged impacts on slip-resistance operations with other types of smooth floors and flooring materials.

Although many causes and different factors were involved in the flooring wear developments, it became evident that variations in the topographic aspects of smooth floors were accompanied by intensive wear progress and accordingly caused to changes on traction performance. Therefore, surface finishes of the floors, especially soft and smooth floors and flooring materials, should be periodically monitored with their slip resistance properties to effectively control and prevent pedestrian fall incidence.

3.2.2. Recognition of Wear Developments. Findings from the surface profile measurements and surface structure observations evidently showed clear proofs of wear progress of the PMMA surfaces after the tests. Figure 6 shows an example of the tested track at a sliding line of a PMMA surface with a roughness profile and a micrographic image. The measured surface profile roughness visibly proved that the surface structure of the rubbed PMMA sample was largely modified after the test. This change was found not only in the first phase of skid-resistance tests between the fresh PMMA samples where the surface roughness was as created by blasting preparations and the three shoes but also in the second phase of skid-resistance tests between the 4 rubbed PMMA specimens, where the surface roughness was evolved by wear behaviours, and the 3 shoes.

Microscopic works also clearly identified robust regional wear developments on the tested PMMA surfaces (Figures 4 and 5). Recurrent regional rubbings seemed to create clean microgrooves instigated by forced distortions and/or microcuttings without any substance relocations. Surface 


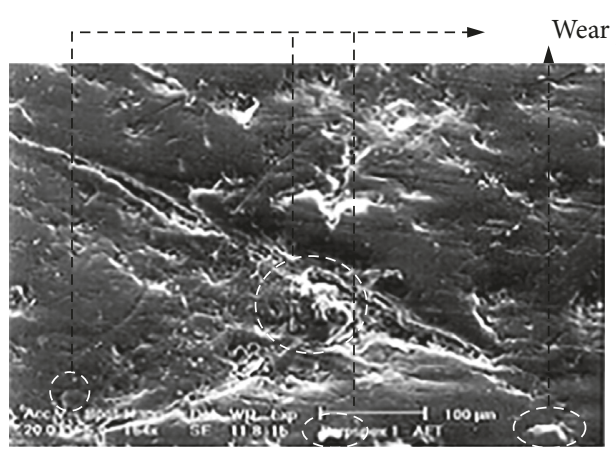

(a)

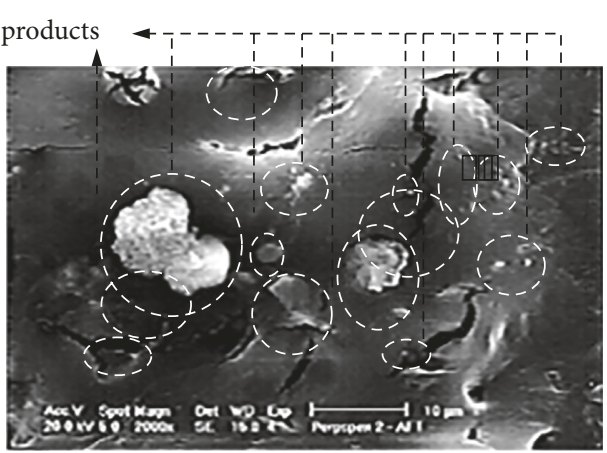

(b)

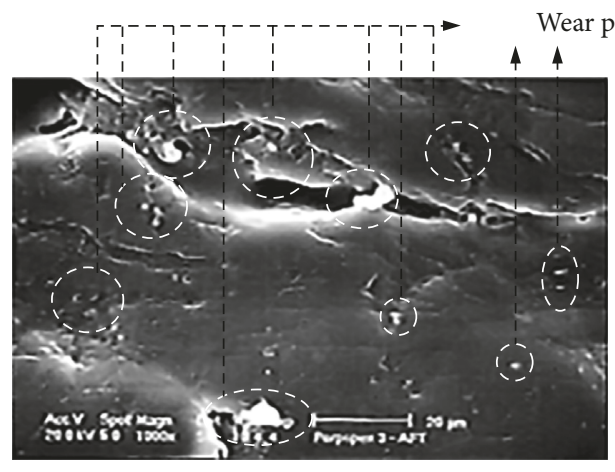

(c)

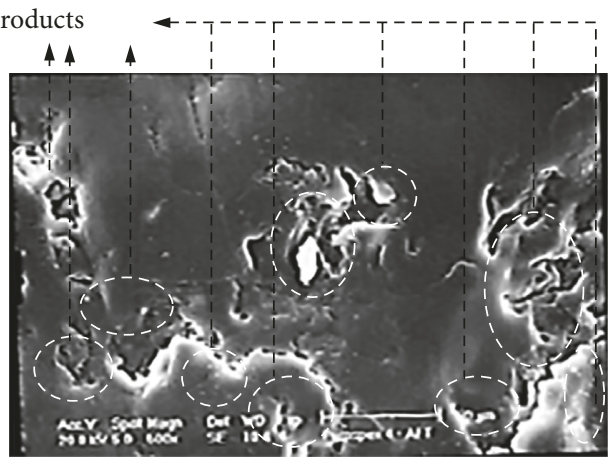

(d)

FIGURE 4: Photoelectron micrographs for the four PMMA surfaces rubbed alongside the three shoes after the dynamic friction tests (ADFT): (a) PMMA1, (b) PMMA2, (c) PMMA3, and (d) PMMA4.

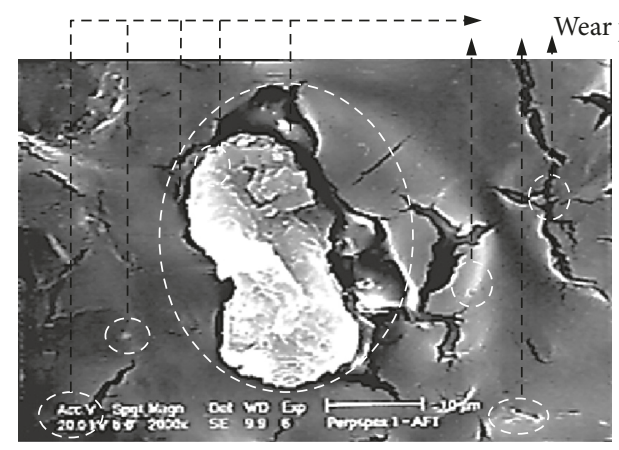

(a)

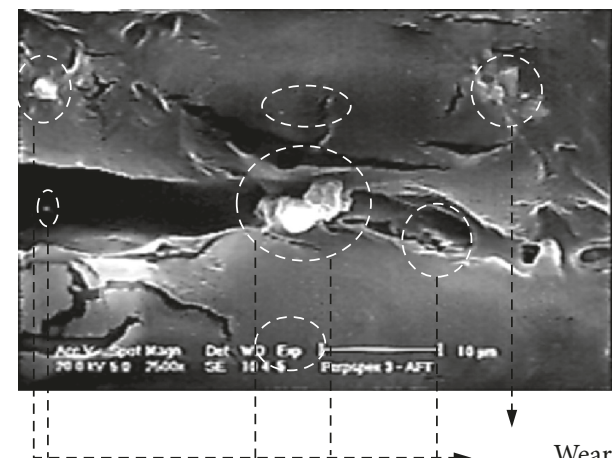

(c)

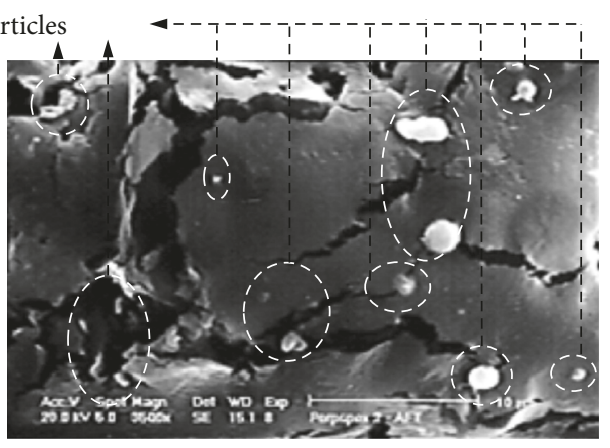

(b)

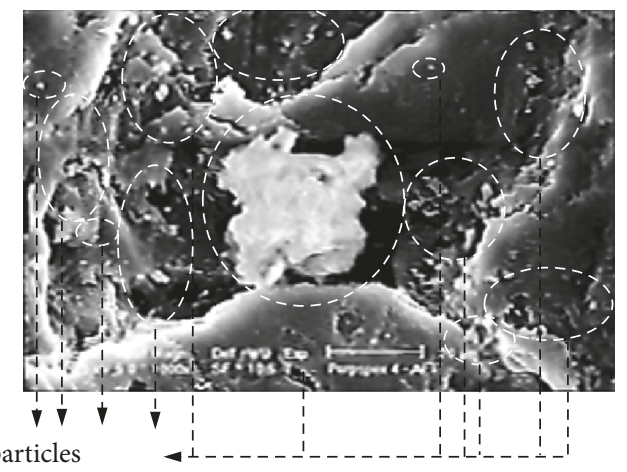

(d)

FIgURe 5: Photoelectron micrographs for the wear particles from rubbed tracks of the PMMA surfaces after the dynamic friction tests (ADFT): (a) PMMA1, (b) PMMA2, (c) PMMA3, and (d) PMMA4. 


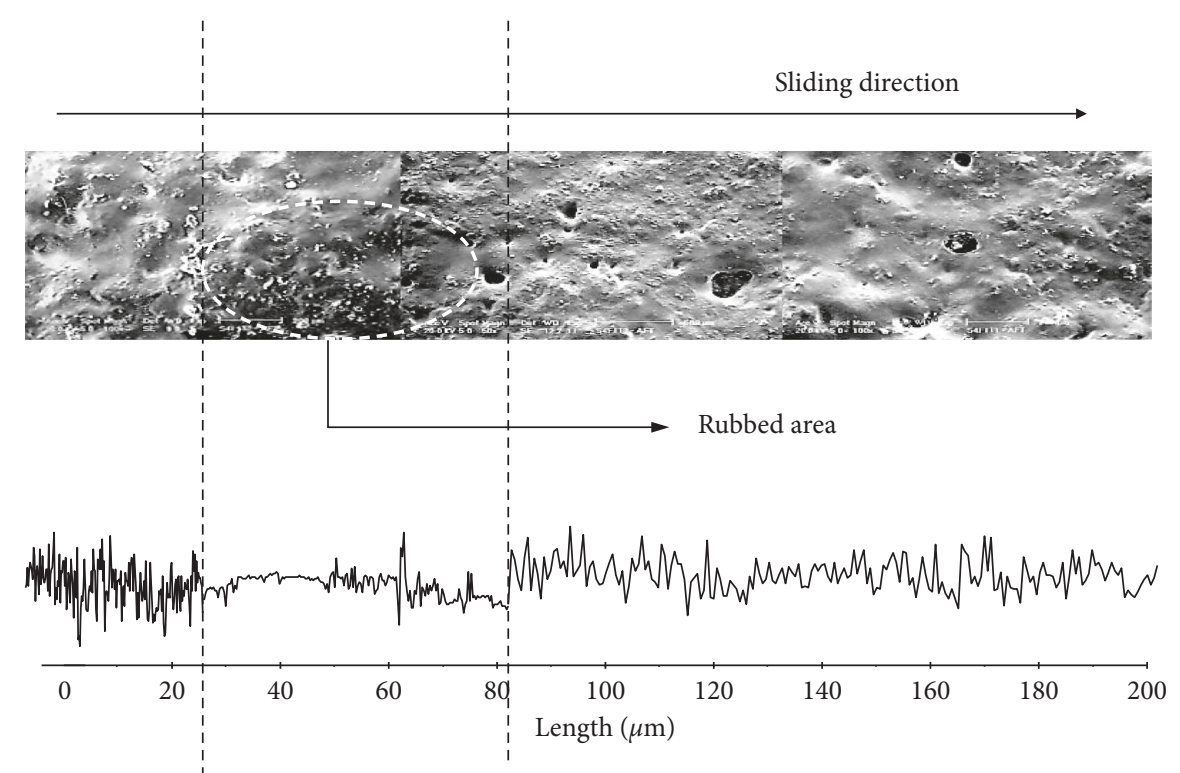

Figure 6: A rubbed track of a PMMA surface with a roughness profile and a photoelectron micrographic image after the dynamic friction tests (ADFT).

asperities of the PMMA samples entered the heel/sole areas and generated wear materials by means of abrasions, adhesion, and plastic deformations [1, 8, 23, 24]. As a result, he tested PMMA surfaces provided increased amounts of contingent proofs on the wear advances that were caused by repetitive asperity contacts directing to localized fatigue failures.

Under such wear-in-progress conditions, further skidding (or walking) could be controlled by buildups of displaced wear elements due to a series of tribophysical events such as abrasion, adhesion, mechanical interconnecting, deformation, and/or a concoction of all those events $[1,8,23,24]$. The moved wear substances would be sheared in the PMMA surfaces along the sliding direction afterwards or possibly during the relocating events $[1,8]$. Consequently, the wear fragments and particles seem to be maligned, compressed, ruptured, and started to merge with additional substances with repeated rubbings $[1,8]$. Hence, different steps of wear advances and topographic alterations seem to be developed concurrently at discrete sites of the boundary areas between the PMMA and shoe surfaces with additional rubbings (or ambulation). This sequence of wear activities appears to absolutely change surface features of the PMMA samples and ultimately skid-resistance properties.

Findings from this study also delivered firm evidence on advanced information for flooring wear mechanisms such as reverse and communal transfers of wear materials as found in the recent studies $[1,8,23,24]$. Figure 7 proposes an aggregate wear model for a floor surface skidding against a shoe heel during frequent walking. The model demonstrates that blasted materials from the PMMA surfaces can be transferred into the shoe heel/sole. Since the relocated wear materials to the shoe surface may instigate microscratches on the PMMA surface, the possible source of wear development seems to be caused by displaced wear products. In this process, the entire shoe-floor skidding system would be operated through freshly transferred wear materials between the PMMA surface and polymeric substances.

The wear particles also seemed to be coherent with such a condition, in which the load was sustained by trifling slices of radically work-hardened substances [1, 8, 23, 24]. This type of wear features should be further examined in the future study since the outcomes can be significantly altered if this would be applied to real-world walking situations where recurrent skidding (walking) is performed at the same trails between the floor surfaces and shoe heels/soles. Therefore, information on flooring wear behaviours, characteristics, and mechanisms would be beneficial for the development of new pedestrian flooring materials and floor surface designs in the future.

\subsubsection{Verification of the Flooring Wear Model. Many new} aspects of flooring wear developments were learnt by observing smooth PMMA surfaces rubbed against three shoes. Flooring wear behaviours were the result of numerous regional interactions against the shoe surfaces so the nature of these events was very complicated to control if they were not investigated at an initial phase of skid-resistance assessments. Although monitoring the surface finishes with roughness parameters was a significant indicator to detect topographic changes of the PMMA specimens, identifying specific surface features responsible for the wear developments of floor surfaces seemed to be regarded as an important first step.

As evidently learned from the findings of this study, wear growths were most likely to inaugurate from the valley areas of floor surfaces. However, this aspect was not fully investigated in the literature. Minor variations in the deep basin areas of the PMMA surfaces seemed to 


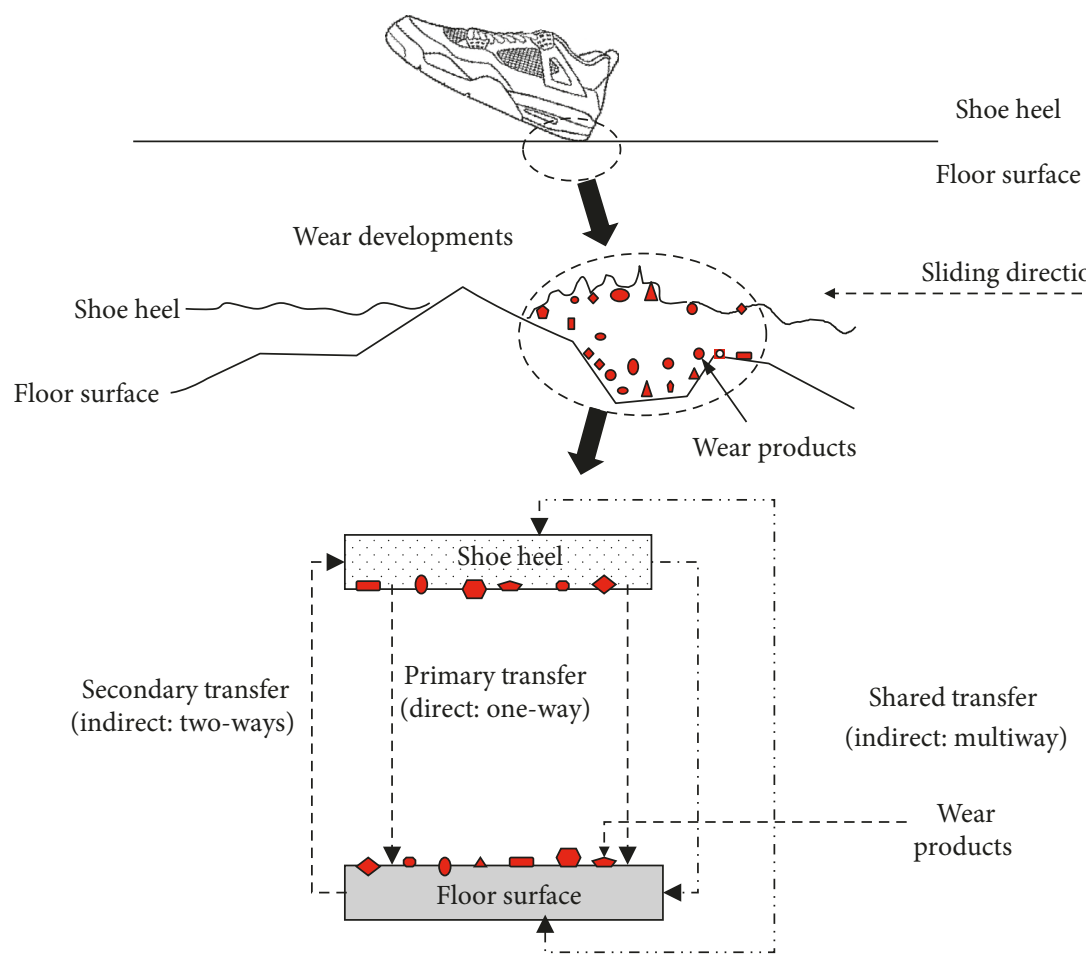

FIGURE 7: A flooring wear model suggests three probable wear mechanisms: primary (one-way), secondary (two-ways), and shared (multiways) wear modes.

considerably affect friction and wear mechanisms without causing many changes to the peak areas of surface asperities. Whilst the PMMA surfaces remain essentially unsystematic in their topographic structures, substantial retreats from randomness might occur in those surface structures which were accountable for wear progress. Thus, a diagnostic technique to segregate those topographical aspects is needed to identify their specific roles to flooring wear behaviours.

As proposed in the recent two studies, a flooring wear concept and model was tested by different types of flooring materials: metals, ceramic, terracotta, and vinyl plates $[1,8]$. The wear model suggested three probable causes of flooring wear systems: primary- (one-state), secondary- (two-state), and shared-wear modes (multistate). This idea of wear evolutions also displayed creations and travels of abraded substances between the floor and shoe surfaces.

Overall outcomes from the current study strongly support that local moving of wear substances from the shoe heels/soles to the PMMA surfaces is an important wear process in the shoe-floor skidding system. When the relocated wear products are formed on the PMMA surfaces during the repeated rubbings, an elastic modulus of the transferred wear products seems to be comparable to their bulk material ones. As a result, the localized stresses between the two bodies are reduced and the contact areas are increased.

These factors seem to mainly affect the wear mechanisms amongst the PMMA surfaces and shoe heels and significantly contribute to lessening wear rates following the development of uniformly transferred wear coatings. Hence, it can be confirmed that the proposed flooring wear concept is evenly imperative to appreciating traction performance and its resultant impacts on wear growths of the floor surfaces. This means that, unlike other mechanical characteristics, wear activities of the floor surface are not inherent but are exclusive to skidding arrangements and adaptable by skidding environments such as counterface topographic features and their interactions [1, 8, 27-30].

\section{Conclusions}

Understanding wear behaviours and mechanisms of floor surfaces with the quantification of slipperiness may allow us to better understand slip-resistance properties, but this issue was not systematically explored. The multifaceted aspects of tribological actions amongst the floors, shoes, and environments require in-depth approaches to provide more accurate assessments and interpretations. In this sense, this study explored friction-induced wear growths of smooth resilient floors and identified their impacts on traction performance.

Laboratory-based skid-resistance tests were conducted between deliberately arranged 4 PMMA samples and 3 shoes. The tested surfaces of each PMMA sample were extensively analyzed by measuring surface profiles and observing surface structures. By understanding wear behaviours and recognizing associated tribophysical characteristics, the present study evidently showed the importance of flooring wear advances and their significant controls on 
skid-resistance operations. The following outcomes were summarized:

(1) Heavy surface changes of the PMMA samples were uncovered not only in the initial stage but also in the subsequent one of skid-resistance tests

(2) The rubbed PMMA specimens were considerably impacted by skid-resistance performance

(3) Amongst the assessed profile roughness parameters, the $R_{\mathrm{vm}}$ parameter (maximum mean depth) of PMMA surfaces experienced the largest variation ( $>50 \%$ on average) after the tests

(4) Large amounts of wear products were accumulated on the tested surface trails, depending upon the formed profile roughness of the PMMA samples

(5) Wear developments of the PMMA surfaces were also progressed by three steps of wear mechanisms: primary- (directly: one-state), secondary- (indirectly: two-state), and shared-wear modes (indirectly: multistate)

(6) Overall results also confirmed that flooring wear behaviours could not be distinguished by a solitary wear form because dissimilar types of wear mechanisms were developed upon not only different parts of surface heights but also different stages of dynamic friction measurements

(7) This study identified specific information on wear behaviours, characteristics, and mechanisms for the smooth resilient floors

(8) Outcomes from this study clearly verified the flooring wear concept and model to offer a future guidance on flooring safety evaluation

(9) This evidence would be advantageous for the design improvements and/or developments of new flooring materials for the pedestrian floorings and footpaths to avert fall incidents

\section{Nomenclature}

ADFT: After the dynamic friction tests

BDFT: Before the dynamic friction tests

DFC: Dynamic friction coefficient

PMMA: Polymethyl methacrylate

PMMA1: PMMA Sheet No. 1

PMMA2: PMMA Sheet No. 2

PMMA3: PMMA Sheet No. 3

PMMA4: PMMA Sheet No. 4

$R_{\mathrm{a}}$ : $\quad$ Centre line average roughness

$R_{\mathrm{q}}$ : $\quad$ Root-mean-square roughness

$R_{\mathrm{tm}}$ : $\quad$ Maximum mean peak-to-valley height

$R_{\mathrm{pm}}$ : $\quad$ Maximum mean peak height

$R_{\mathrm{vm}}$ : $\quad$ Maximum mean depth height

$R_{\mathrm{sk}}$ : $\quad$ Skewness

$R_{\mathrm{kr}}$ : Kurtosis

$\Delta_{\mathrm{a}}: \quad$ Average slope angle

SEM: $\quad$ Scanning electron microscope.

\section{Data Availability}

The data that support the findings of this study are available from the corresponding author, I. J. Kim, upon reasonable request.

\section{Conflicts of Interest}

The author declares that there are no conflicts of interest regarding the publication of this article.

\section{Acknowledgments}

This study was supported by a research project grant (Grant No. 1702040565-P) from the University of Sharjah, Sharjah, UAE.

\section{References}

[1] I. J. Kim, "A study on wear development of floor surfaces: impact on pedestrian walkway slip-resistance performance," Tribology International, vol. 95, pp. 316-323, 2016.

[2] S. K. Choi, R. Kudoh, J. Koga et al., "A comparative evaluation of floor slip resistance test methods," Construction and Building Materials, vol. 94, pp. 737-745, 2015.

[3] Resilient Floor Covering Institute (RFCI), "Resilient flooring products," 2015, http://rfci.com/about-us/.

[4] Flooring Industry Council (FIC), Flooring Materials-Resilient, Flooring Industry Council, St. Louis, MO, USA, 2015, http:// www.ficstl.org/Flooring-Materials/Resilient.

[5] Vinyl Council of Australia (VCA), "PVC building solutions," 2015, http://www.vinyl.org.au/pvc-in-building/pvc-buildingproducts.

[6] S. Leclercq and H. Saulnier, "Floor slip resistance changes in food sector workshops: prevailing role played by "fouling"," Safety Science, vol. 40, no. 7-8, pp. 659-673, 2002.

[7] I. J. Kim, H. Hsiao, and P. Simeonov, "Functional levels of floor surface roughness for the prevention of slips and falls: clean-and-dry and soapsuds-covered wet surfaces," Applied Ergonomics, vol. 44, no. 1, pp. 58-64, 2013.

[8] I. J. Kim, "Investigation and interpretation of flooring wear developments for pedestrian fall safety assessments," Tribology Transactions, vol. 61, no. 1, pp. 168-177, 2018.

[9] I. J. Kim,"A practical design search for optimal floor surface finishes," in Pedestrian Fall Safety Assessments: Improved Understanding on Slip Resistance Measurements and Investigations, pp. 199-224, Springer International Publishing AG, Springer International Publishing AG, Cham, Switzerland, Chapter 7, 2017.

[10] I. J. Kim, "Investigation of floor surface finishes for optimal slip resistance performance," Safety and Health at Work, vol. 9, no. 1, pp. 17-24, 2017.

[11] L. Strandberg, "On accident analysis and slip-resistance measurement," Ergonomics, vol. 26, no. 1, pp. 11-32, 1983.

[12] M. S. Redfern and B. Bidanda, "Slip resistance of the shoefloor-interface under biomechanically-relevant conditions," Ergonomics, vol. 37, no. 3, pp. 511-524, 1994.

[13] W. R. Chang and S. Matz, "The slip resistance of common footwear materials measured with two slipmeters," Applied Ergonomics, vol. 32, no. 6, pp. 549-558, 2001.

[14] K. W. Li, H. H. Wu, and Y. C. Lin, "The effect of shoe sole tread groove depth on the friction coefficient with different tread groove widths, floors and contaminants," Applied Ergonomics, vol. 37, no. 6, pp. 743-748, 2006. 
[15] L. Liu, K. W. Li, Y. H. Lee, C. C Chen, and C. Y. Chen, "Friction measurements on "anti-slip" floors under shoe sole, contamination, and inclination conditions," Safety Science, vol. 48, no. 10, pp. 1321-1326, 2010.

[16] P. L. Menezes, Kishore, S. V. Kailas, and M. R. Lovell, "Friction and transfer layer formation in polymer-steel tribosystem: role of surface texture and roughness parameters," Wear, vol. 271, no. 9-10, pp. 2213-2221, 2011.

[17] M. J. H. Cowap, S. R. M. Moghaddam, P. L. Menezes, and K. E. Beschorner, "Contributions of adhesion and hysteresis to coefficient of friction between shoe and floor surfaces: effects of floor roughness and sliding speed," TribologyMaterials, Surfaces \& Interfaces, vol. 9, no. 2, pp. 77-84, 2015.

[18] S. Derler, F. Kausch, and R. Huber, "Analysis of factors influencing the friction coefficients of shoe sole materials," Safety Science, vol. 46, no. 5, pp. 822-832, 2008.

[19] W. R. Chang, T. K. Courtney, R. Grönqvist, and M. S. Redfern, "Measuring slipperiness-discussions on the state of the art and future research," in Measuring Slipperiness: Human Locomotion and Surface Factors, W. R. Chang and T. K. Courtney, Eds., Taylor \& Francis, London, UK, 2003.

[20] K. W. Li, W. R. Chang, T. B. Leamon, and C. J. Chen, "Floor slipperiness measurement: friction coefficient, roughness of floors, and subjective perception under spillage conditions," Safety Science, vol. 42, no. 6, pp. 547-565, 2004.

[21] W. R. Chang, Y. H. Huang, K. W. Li, A. Filiaggi, and T. K. Courtney, "Assessing slipperiness in fast-food restaurants in the USA using friction variation, friction level and perception rating," Applied Ergonomics, vol. 39, no. 3, pp. 359-367, 2008.

[22] Bio-Rad, MRC-600, Confocal Imaging System Operating Manual, Bio-Rad Microscience Division, Watford, UK, 1988.

[23] I. J. Kim, "Wear observation of shoe surfaces: application for slip and fall safety assessments," Tribology Transactions, vol. 58, no. 3, pp. 407-417, 2015.

[24] I. J. Kim, "Identifying shoe wear mechanisms and associated tribological characteristics: the importance for slip resistance evaluation," Wear, vol. 360-361, pp. 77-86, 2016.

[25] British Standards Institution (BSI), BS 1134: Assessment of Surface Texture: Part 1. Methods and Instrumentation, British Standards Institution, London, UK, 1988.

[26] International Organization for Standardization (ISO), Geometrical Product Specifications (GPS): Surface Texture: Profile Method-Terms, Definitions and Surface Texture Parameters, ISO 4287, ISO, Geneva, Switzerland, 1998, http://isotc213.ds. dk/jgohf/main/c-4th\%20plenary\%20meeting/213N159.pdf.

[27] S. Bahadur, D. Gong, and J. Anderegg, "Investigation of the influence of $\mathrm{CaS}, \mathrm{CaO}$, and $\mathrm{CaF}_{2}$ fillers on the transfer and wear of nylon by microscopy and XPS analysis," Wear, vol. 197, no. 1-2, pp. 271-279, 1996.

[28] L. Yu and S. Bahadur, "An investigation of the transfer film characteristics and the tribological behavior of polyphenylene sulfide composites in sliding against tool steel," Wear, vol. 214, no. 2, pp. 245-251, 1998.

[29] Q. Zhao and S. Bahadur, "A study of the modification of the friction and wear behavior of polyphenylene sulfide by particulate Ag2S and PbTe fillers," Wear, vol. 217, no. 1, pp. 62-72, 1998.

[30] Q. Zhao and S. Bahadur, "The mechanism of filler action and the criterion of filler selection for reducing wear," Wear, vol. 225-229, pp. 660-668, 1999. 


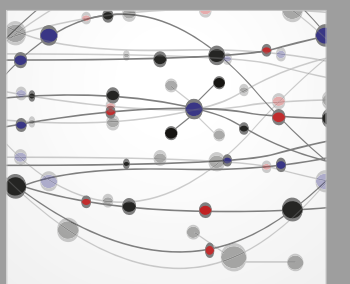

The Scientific World Journal
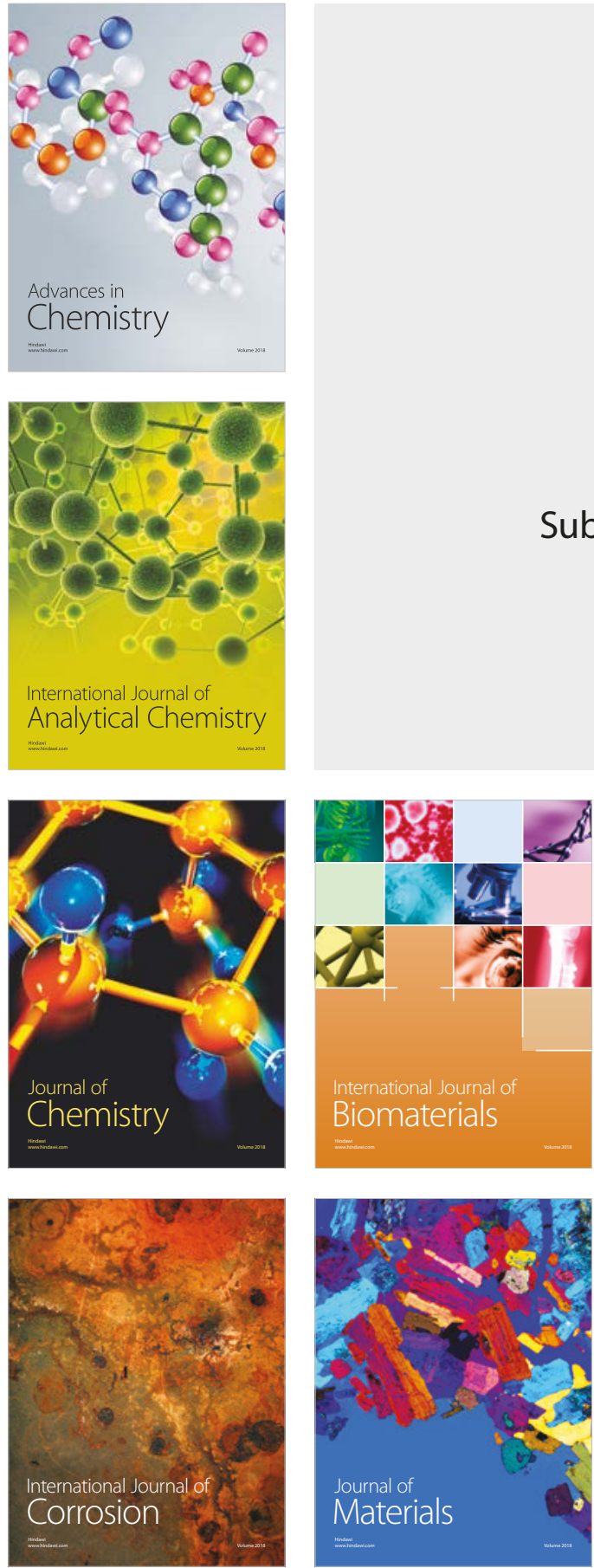

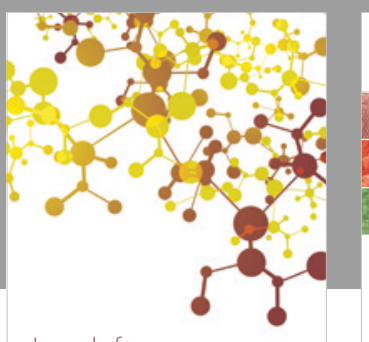

Journal of

Applied Chemistry
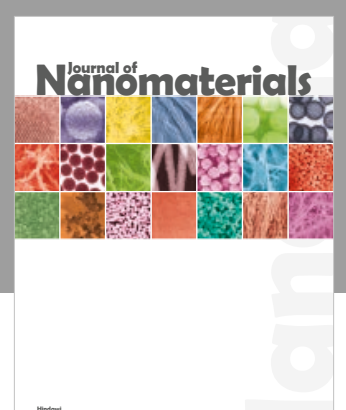

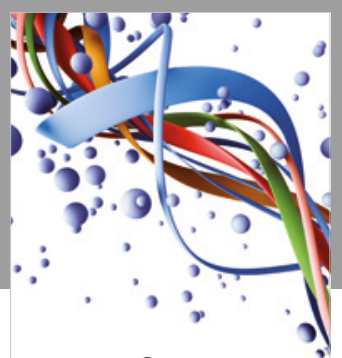

Scientifica

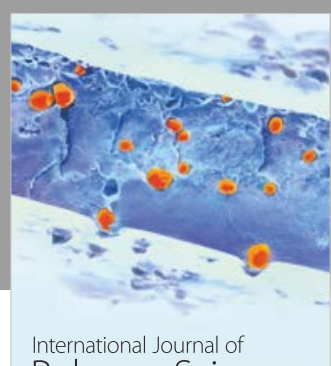

Polymer Science

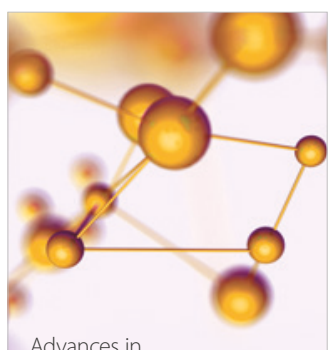

Physical Chemistry
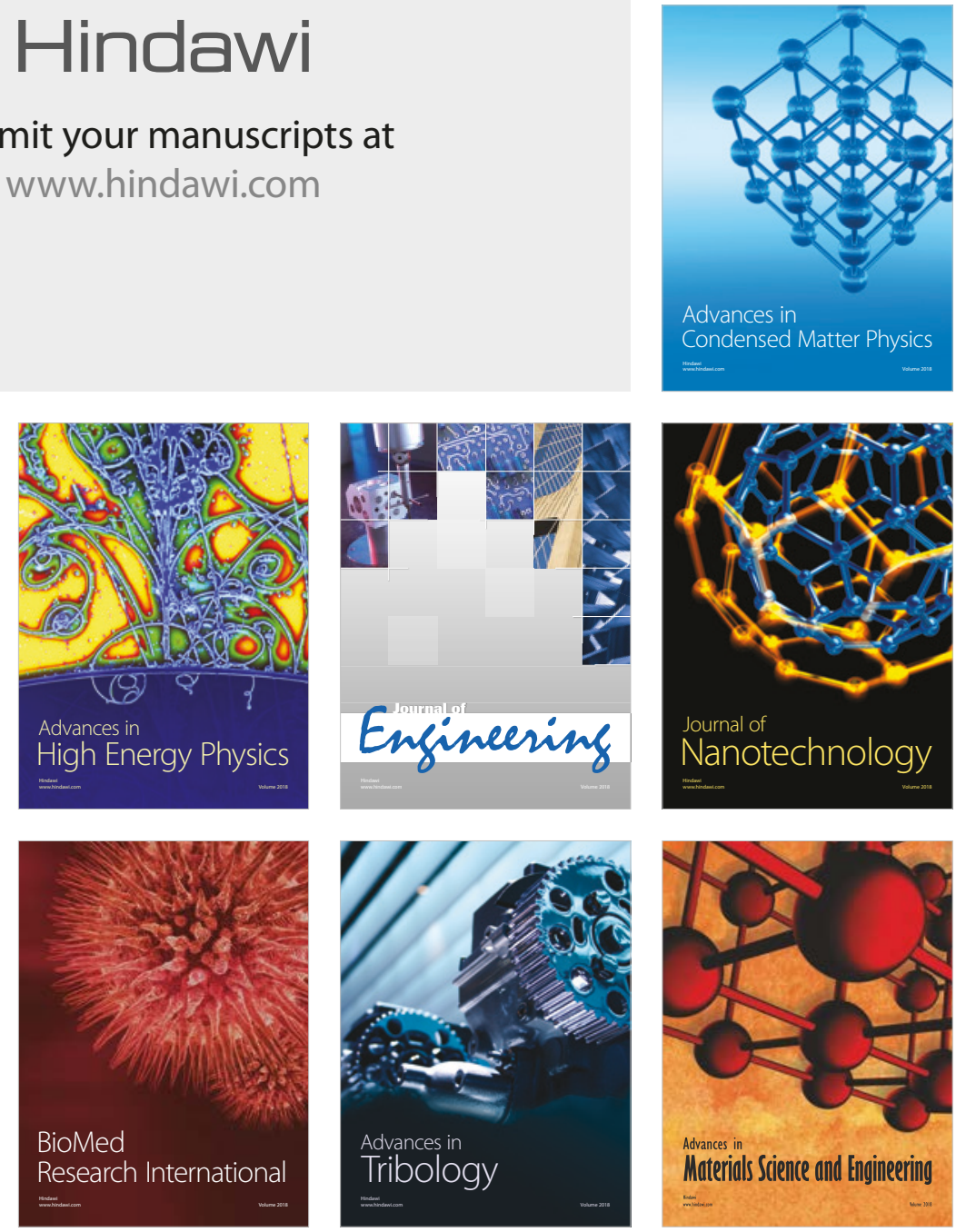